\title{
Lipid mediators of inflammation in rheumatoid arthritis and osteoarthritis
}

\author{
Hilde Brouwers, Joost von Hegedus, René Toes, \\ Margreet Kloppenburg, Andreea Ioan-Facsinay \\ Dept. of Rheumatology, Albinusdreef 2, 2333 ZA, Leiden University Medical Center, Leiden, The Netherlands
}

\section{Keywords:}

Osteoarthritis

Rheumatoid arthritis

Lipid mediators

Lipids

Inflammation

Oxylipins

\begin{abstract}
A B S T R A C T
Rheumatoid arthritis (RA) and osteoarthritis (OA) are inflammatory joint diseases, characterized by pain and structural damage. Besides prostaglandins, usually targeted by non-steroidal anti-inflammatory drugs, other lipids, including fatty acids, phospholipids and other bioactive lipid mediators derived from fatty acids could also contribute to RA and OA.

In this review, we present evidence for the role of fatty acids and derivatives in RA and OA by summarizing findings related to their presence in serum and synovial fluid, as well as their association with clinical characteristics and effects on RA and OA tissues in vitro. Finally, a more direct evidence for their role in RA and OA derived from intervention studies in humans or mouse models of disease is summarized. Based on the presented data, we present a research agenda, in which some key unresolved questions regarding the role of lipids in RA and $\mathrm{OA}$ are formulated.
\end{abstract}

๑) 2016 Elsevier Ltd. All rights reserved.

\section{Introduction}

Rheumatoid arthritis (RA) and osteoarthritis (OA) are joint diseases characterized by different pathophysiological mechanisms, but displaying common clinical characteristics, such as joint pain, functional impairment and structural damage which is hallmarked by bone erosions in RA and

\footnotetext{
* Corresponding author. Tel.: +3171 5263598; fax: +31 715266752.

E-mail addresses: H.Brouwers@lumc.nl (H. Brouwers), J.H.von_Hegedus@lumc.nl (J. von Hegedus), R.E.M.toes@lumc.nl (R. Toes), G.Kloppenburg@lumc.nl (M. Kloppenburg), A.Ioan@lumc.nl (A. Ioan-Facsinay).
} 
osteophytes in OA. Moreover, both diseases display joint space narrowing, reflecting cartilage loss. Another common feature of these diseases is the presence of inflammation in the majority of the patients. While the role of inflammation in the pathogenesis of RA has been established previously, its presence and possible role in OA has been only recently revealed. Several studies during the past 10 years have shown an association between synovial inflammation and pain on one hand and radiographic progression on the other hand, thus establishing the pivotal role of inflammation in OA (reviewed in Refs. [1,2]).

Fatty acids acquired through diet are usually transported through the body in triglycerides or phospholipids incorporated in lipoproteins, but can also be found in free form in blood. Moreover, they are present both in bound and free form in cells, where they perform various functions as energy source, membrane constituents or signalling molecules. They are the essential building blocks for higher-order lipids such as phospholipids, sphingolipids, glycerolipids and glycolipids. Moreover, they could be metabolized into bioactive lipid mediators such as oxylipins, including eicosanoids (prostaglandins, thromboxanes and leukotrienes) and other lipids with more anti-inflammatory and proresolving activity such as lipoxins, resolvins, maresins and protectins. Enzymes such as phospholipases (PLAs) which release fatty acids from phospholipids, cyclooxygenases (COXs) and lipoxygenases (LOXs) that oxidize fatty acids are involved in the generation of oxylipins. Fatty acids, higher-order lipids and oxylipins can interact with inflammatory as well as tissue-resident cells, thereby contributing to various processes in the body, including inflammation, wound healing, pain, etc, and potentially playing a role in RA and OA. In general, it is believed that saturated fatty acids, n-6 polyunsaturated fatty acids (PUFAs), such as arachidonic acid (AA), and AA derivatives (prostaglandins and leukotrienes) have a pro-inflammatory effect. By contrast, unsaturated fatty acids, n-3 PUFA, such as docosahexaenoic acid (DHA) and eicosapentaenoic acid (EPA), and the oxylipins derived from them (resolvins, maresins and protectins) are believed to have an anti-inflammatory function. The latter are also believed to be pro-resolving, thereby actively helping wound healing and return to tissue homeostasis after an inflammatory response.

In this review, we will present data supporting the role of lipids in RA and OA. To this end, we will summarize findings from three lines of evidence. First, data related to the presence of various fatty acids and their derivatives (lipid mediators) in blood or synovial fluid (SF) of RA and OA patients will be summarized. Next, the association of these lipids with clinical disease parameters will be presented, as well as in vivo intervention studies both in humans and mouse models of disease. Finally, in vitro studies indicating the effects of these lipids on human joint tissues will be presented (summarized in Fig. 1). The role of cholesterol and lipoprotein metabolism in RA and OA has been reviewed elsewhere $[3,4]$ and will not be discussed in this review.

Lipids and lipid classes described in serum of healthy individuals

In an extensive study in which the major six lipid categories as defined by the Lipid Maps were measured in plasma of healthy individuals, more than 500 lipid species were identified [5]. The measured sample was obtained from the National Institute of Standards and Technology (USA); the pooled plasma sample was obtained and stored after overnight fasting in a standardized fashion, from 100 healthy individuals (age: between 40 and 50 years) including an equal number of men and women whose ethnicity was representative of the US population. The most abundant (on a molar basis) was sterols (including cholesterol), followed by triglycerides (part of lipoproteins), glycerophospholipids, free fatty acyls and sphingolipids, and the least abundant was diacylglycerols and prenols. In terms of free fatty acids, oleic acid, followed by palmitic acid and stearic acid were the most abundant and comprised approximately $78 \%$ of all free fatty acids after overnight fasting. The most abundant PUFA were linoleic acid (LA) and AA, but EPA and DHA, which are derived from fish oil and are known for their anti-inflammatory effects, were also detectable. Lipid mediators such as oxylipins were also detected in plasma, with 15 -deoxy- $\mathrm{PGD}_{2}$ (prostaglandin $\mathrm{D} 2$ ) being the major metabolite generated by COX, while 5-HETE was the most prominent eicosanoid of the LOX pathway found in plasma [5].

This review focuses on selected lipids in the six lipid classes: fatty acids either in free form or incorporated in higher-order lipids (especially phospholipids), as well as their bioactive lipid mediators in RA and OA (Table 1). 
A

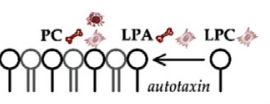

Higher order lipids

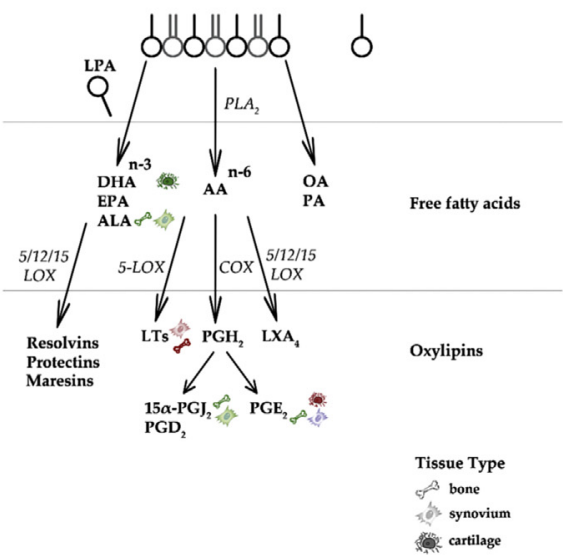

B

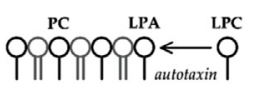

Higher order lipids

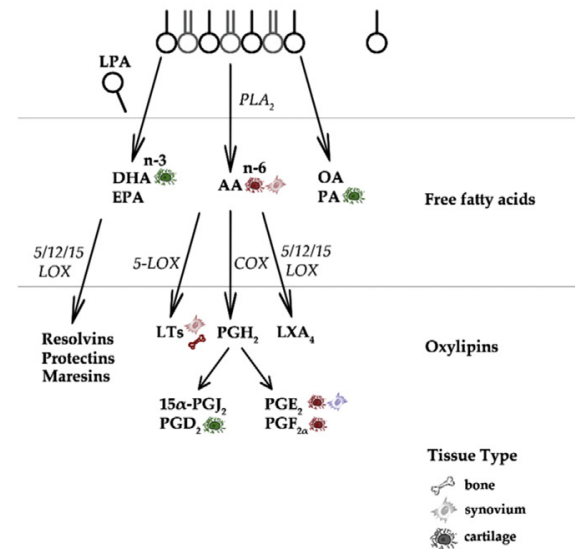

Fig. 1. Summary showing lipids and relevant enzymes (in italic) in the context of rheumatoid arthritis (A) or osteoarthritis (B) discussed in this review. Lipids are incorporated in phospholipids in the membrane. The enzyme autotaxin can convert lysosphosphatidylcholine (LPC) to lysophospatidic acid (LPA), and phopholipases (PLA) can both generate LPC from phosphatidylcholine (PC) and release fatty acids from the membrane phospholipids. These fatty acids can be metabolized into oxylipins by different cyclooxygenases (COX) and lipoxygenases (LOX). Symbols represent different tissues that have been shown to be affected by lipids. The effects of lipids on tissues are indicated by colours: red $=$ inflammatory, green $=$ anti-inflammatory/resolving, blue $=$ both inflammatory and anti-inflammatory/resolving.

\section{Presence of fatty acids and derivatives in RA and $\mathrm{OA}$ patients}

\section{Lipids in serum of RA patients}

Direct comparisons between different lipids in RA serum/plasma and healthy controls were only made in few studies and generally included relatively low numbers of patients (between 10 and 16). In a metabolomics study, it was found that the levels of lipids in general were lower in serum of newly presenting RA patients compared to healthy controls although a systematic investigation of different lipid classes was not performed. To exclude the effect of disease-modifying anti-rheumatic drugs (DMARDs) on metabolic status, patients who were administered DMARDs were excluded from this analysis [6]. In another study in 166 RA patients, the levels of free fatty acids were found to be similar in RA patients and healthy controls [7], indicating that free fatty acids are not quantitatively different in the diseased state. Fatty acids in phospholipids and sphingomyelins were also described in serum of healthy individuals and RA patients [8]. Of the different phospholipids studied, only the ratio phosphatidylcholine (PC)/lysophosphatidylcholine (LPC, generated from PC by the PLA enzyme) was found to be lower in serum of RA patients compared to healthy individuals [9], indicating a higher activation of PLA in RA patients. This could result in higher levels of free fatty acids that can further be metabolized into bioactive lipids. Indeed, lipid mediators such as prostaglandins, $\mathrm{PGD}_{2}$ and $\mathrm{PGE}_{2}$ [10], generated by COX enzymes, were shown to be present in serum of RA patients, indicating that not only precursor fatty acids might be higher in RA compared to healthy individuals, but also certain enzymes involved in generation of bioactive lipids. However, bioactive lipids were mainly studied in SF.

\section{Lipids in SF of RA patients}

Broad lipidomic studies in SF of RA patients were performed in which most of the lipid species described in serum/plasma could be detected [8,10-13]; several phospholipid and fatty acid species 


\section{Table 1}

Summary of lipids included in the present review and their corresponding lipid classes. DHA: docosahexaenoic acid; EPA: eicosapentaenoic acid; PC: phosphatidylcholine; LPC: lysophosphatidylcholine; LPA: lysophospatidic acid.

\begin{tabular}{ll}
\hline $\begin{array}{l}\text { Lipid class } \\
\text { (according to Lipid Maps) }\end{array}$ & $\begin{array}{l}\text { Lipids included } \\
\text { in this review }\end{array}$ \\
\hline Fatty acyls & $\begin{array}{l}\text { Fatty acids, oxylipins: eicosanoids } \\
\text { (prostaglandins, leukotrienes, lipoxins), } \\
\text { derivatives of DHA, EPA (resolvins, maresins, protectins) }\end{array}$ \\
Glycerolipids & - \\
Glycerophospholipids & Phospholipids (e.g. PC) and lysophospholipids (e.g. LPC, LPA) \\
Sphingolipids & sphingomyelin \\
Sterol lipids & - \\
Prenol lipids & - \\
\hline
\end{tabular}

were found. Similarly to plasma, PC were the most abundant phospholipids in SF, followed by LPC and sphingomyelins [12] and their levels were higher in RA SF than controls [12,13]. The ratio of PC/LPC was higher in RA SF than in controls, in contrast to what was found in serum [12,13]. A detailed analyses of the species of lipids revealed that RA SF was relatively enriched in LPC-containing saturated fatty acids, while saturated PC was lower than in controls, indicating that saturated fatty acids were possibly more efficiently released from PC in RA. The length of fatty acids contained in PC species was not different between RA SF and controls, but RA SF contained relatively more short-chain fatty acids in LPC compared to controls $[12,13]$. As a longer chain length and higher saturation grade are believed to be beneficial for the lubricating properties of phospholipids, these data could indicate that the phospholipids present in RA SF are less potent lubricators and provide less protection against mechanical damage than in healthy controls. All studies were small and included approximately 20 patients.

Eicosanoids such as prostaglandins and leukotrienes were intensively investigated in SF of RA patients and were recently reviewed [14]. Prostaglandin E2 $\left(\mathrm{PGE}_{2}\right)[14,15]$ as well as PLA 2 and COX, enzymes involved in its generation, were found to be higher in SF or were higher expressed in synovium of RA patients (mPGES) [16-20]. Likewise, the enzyme converting $\mathrm{PGE}_{2}$ into the inactive 15-PGDH was higher in RA synovium, indicating that regulatory mechanisms targeting $\mathrm{PGE}_{2}$ are also activated in this disease. Interestingly and in line with this observation, the more anti-inflammatory prostaglandins, $\mathrm{PGD}_{2}$ and its metabolite 15-deoxy-PGJ 2 were also found in the RA joint. Expression of $\mathrm{PGD}_{2}$ was detected in cells in RA joints; however, no evidence was found for enhanced biosynthesis of 15-deoxy$\mathrm{PGJ}_{2}$. Next to prostaglandins, leukotrienes such as $\mathrm{LTB}_{4}, \mathrm{LTD}_{4}$ and $\mathrm{LTE}_{4}$ were found in SF of RA patients [14].

Interestingly, anti-inflammatory products of LOXs were also described in SF of RA patients. These included the anti-inflammatory and pro-resolving mediators lipoxin A4 ( $\left.\mathrm{LXA}_{4}\right)$ and the mediators derived from the n-3 PUFA DHA: maresin 1 (Mar1) and resolving D5 (RVD5) [11]. Although this study was performed in only five patients and the effects of pro-resolving lipids on RA development or progression were not yet investigated, their potent immune modulatory functions [21] identify them as promising therapeutic agents for chronic inflammatory diseases.

Besides the PLA, COX and LOX enzymes, autotaxin makes an important contribution to bioactive lipids in RA. It can convert membrane phospholipids such as LPC to lysophospatidic acid (LPA), as well as sphingosylphosphorylcholine to yield sphingosine-1-phosphate (S1P). S1P can act as an intracellular second messenger or extracellular lipid mediator via G-coupled receptors and is involved in regulation of pro-inflammatory pathways and cell migration. Its possible role in RA is discussed later. Both LPA and S1P, as well as autotaxin were shown to be present in SF of RA patients [22-24], while higher autotaxin levels were shown in RA SF $(n=16)$ and serum $(n=26)$ compared to controls [25].

\section{Lipids in serum/SF of $O A$}

Several lipidomic studies in OA patients have been performed over the recent years. Although most of them study SF, one study investigated the phospholipid profile of hip and knee OA patients $(n=59$ women) in plasma [26]. Similarly to RA, the LPC/PC ratio was higher in both mild and moderate OA 
(based on radiographic damage) compared to controls, contradictory to that found in SF. In two studies with 30 and 48 OA patients, the levels of phospholipids were found to be elevated in SF of OA patients compared to post-mortem controls [12,13], similar to what was found for RA.

Few studies have investigated oxylipin levels in OA. In one study with $10 \mathrm{OA}$ patients, 8 -iso-PGF 2 . and 15-keto-dihydroPGF $2 \alpha$ were detected in both serum and SF, but the levels did not correlate in these two fluids [27]. This indicates that there might be local production of these lipids, rather than systemic diffusion. Noteworthy is that levels of both metabolites were higher in serum of OA patients compared to controls. Higher levels of these metabolites in serum of OA patients could indicate higher systemic oxidative damage and inflammatory responses. For some patients, the levels of both compounds, but especially 15 -keto-dihydroPGF $2 \alpha$ tended to be somewhat higher in SF compared to serum. It is difficult to interpret these data due to the lack of healthy SF and low numbers of patients. More recently, a study investigated the 15-LOX product 15-HETE and the COX product PGE $_{2}$ in plasma of symptomatic OA patients and controls [28]. Both metabolites were elevated in patients (three different cohorts with 291 patients in total) compared to controls, which could be a systemic reflection of local inflammation in the knee. Similarly, leukotriene $\mathrm{B}_{4}\left(\mathrm{LTB}_{4}\right)$ has also been described in SF of some OA patients, although no comparison with healthy controls was performed [29]. Together, these data indicate that proinflammatory lipids are present in OA patients, although a more extensive investigation of the presence of other lipid classes, such as prostaglandins and anti-inflammatory oxylipins, in OA patients is warranted.

\section{Fatty acids and phospholipids}

Dietary fatty acids, such as the n-3 PUFA EPA and DHA derived from fish oil. These n-3 PUFAs are believed to have anti-inflammatory properties, while the n-6 PUFA AA is believed to be more proinflammatory. Long-chain fatty acids such as AA, EPA and DHA are incorporated in phospholipids and these are essential constituents of membranes. In healthy individuals on a typical Western diet, about $10-20 \%$ of phospholipids in membranes of leukocytes are composed of AA, while $0.5-1 \%$ is EPA and 1.5-3\% is DHA [30]. Dietary intake of EPA and DHA leads to an increase of these fatty acids in cellular membranes and this occurs usually at the expense of AA. This incorporation begins within days and is dose dependent [31,32]. Because erythrocytes have a lifespan of 100-120 days, their membrane phospholipid composition is generally used to monitor the dietary intake of lipids [32,33].

\section{Association with clinical characteristics and intervention studies in RA}

Most studies investigating the types of fatty acids present in RA patients and association with clinical parameters focussed on dietary fatty acids.

Several clinical trials have been performed with either fish oil or n-3 supplementation in RA patients. These randomized control trials have been recently summarized in a systematic review by Miles and Calder [31]. The authors analysed 23 studies, in which EPA and DHA doses varied largely, between $<1$ and $>7 \mathrm{~g} /$ day and were administered mostly orally as fish oil supplements. The duration of the studies varied between 4 and 52 weeks and the placebo controls were usually other types of oils, such as corn, olive oil and paraffin oil. The sample size of these studies was typically around 20-30 patients/ group, excepting few studies with participation of less or more patients. Many studies had methodological shortcomings and no meta-analysis was performed. In general, the observed beneficial effects of n-3 PUFA were related to morning stiffness, the number of tender/swollen joints, grip strength, pain or disease activity. The effects were overall modest.

Since this systematic review, another study investigated the effect of intake of moderate amounts of $\mathrm{n}-3$ PUFA (2.090 g EPA and $1.165 \mathrm{~g}$ DHA) in combination with regular anti-inflammatory therapy in 109 RA patients. High-oleic acid sunflower oil was used as control. Although there was an increase in n-3 PUFA and a relative decrease in n-6 PUFA AA in erythrocyte membranes in the treatment group, there was no significant effect on clinical symptoms, non-steroidal anti-inflammatory drug (NSAID) usage, cytokines, eicosanoids and bone turnover markers in this group [34], which might be attributable to the low dose of n-3 PUFA administered. Similar results regarding the incorporation of n-3 PUFA in erythrocyte membranes at the expense of AA were found in another study with a relative low number 
of patients (54 RA and six psoriatic arthritis patients) in which patients were randomized into three groups: one treated with $3 \mathrm{~g}$ n-3 PUFA/day, one with $3.2 \mathrm{~g} \gamma$-linolenic acid (n-6 PUFA)/day and one with a combination of a low dose n-3 PUFA ( $1.6 \mathrm{~g})$ and $\gamma$-linolenic acid $(1.8 \mathrm{~g}) /$ day. The treatment duration was 12 weeks. A relative decrease of AA/EPA ratio was observed in the n-3 PUFA-treated group, similar to earlier studies. In addition, an enrichment in $\gamma$-linolenic acid in plasma lipids, cholesterol membranes and erythrocyte membranes was observed, and this enrichment was dependent on the dose indicating that this n-6 PUFA is also dose-dependently incorporated in cellular membranes upon intake [35].

Interestingly, several recent studies have investigated the effects of n-3 PUFA on early RA patients or on development of RA and related features in pre-RA individuals. Early RA patients $(n=140)$ treated with DMARD therapy were additionally treated with $5.5 \mathrm{~g} /$ day EPA + DHA or with $0.4 \mathrm{~g} /$ day EPA and DHA (control group). The primary outcome was failure on triple DMARD after 3 months. Failure was lower and the rate of remission was higher in the n-3 PUFA-treated group compared to the control group after 3 months of treatment [36]. Remarkably, EPA in plasma phospholipids was inversely associated with time to remission and to DMARD failure, while similar results, albeit not significant, were also obtained for DHA, indicating that EPA might be more effective than DHA as disease modulator.

Several studies investigated the effect of intake of fish containing n-3 PUFA on the risk of development of RA in healthy individuals. In a meta-analysis involving both prospective and case-control studies, the authors found that for each serving of fish per week, the relative risk of RA became 0.96 (95\% confidence interval $(\mathrm{CI}) 0.91 ; 1.01)$, while there was a $20-24 \%$ lower risk $(0.76(95 \% \mathrm{CI} 0.57 ; 1.02)$ ) of RA for 1-3 servings of fish/week compared to no servings [37]. In an observational population-based cohort study (32,232 women aged 54-89 years), self-reported intake of $>0.21 \mathrm{~g} /$ day of $n-3$ PUFA led to a 35\% (95\% CI 10-52\%) lower risk of developing RA than lower intake. Moreover, long-term consistent intake of $>0.21 \mathrm{~g} /$ day of n-3 PUFA led to a $52 \%$ (95\% CI 29-67\%) lower risk of RA than lower intake, while long-term consistent intake of $>1$ serving of fish/week led to $29 \%$ lower risk (risk ratio (RR) 0.71 ; 95\% CI 0.48-1.04) compared to $<1$ serving/week [38]. Although the intake was based on self-reported data and no measurements of n-3 PUFA in phospholipids were presented, these studies suggest that n3 PUFA could be beneficial for lowering the risk of RA development in pre-disease individuals and this effect is probably dose dependent. This would also be in line with a more recent study in which individuals at risk for developing RA (non-diseased first-degree relatives of RA patients and HLA-DR4+ individuals) and positive for anti-CCP antibodies $(n=30)$ were compared to seronegative individuals ( $n=47$ ) for the amount of n-3 PUFA intake (self-reported) and the percentage of n-3 PUFA in erythrocyte membranes. The findings indicate that anti-CCP ${ }^{+}$individuals were less likely to report n-3 PUFA intake, and the anti-CCP positivity was inversely correlated to the percentage of n-3 PUFA in erythrocyte membranes [39]. Altogether, these findings suggest that n-3 PUFA might affect the risk for development of RA, as well as the clinical parameters of the disease in RA patients.

Supporting these data, interventions with DHA and EPA have been also shown to reduce the onset, incidence and severity of collagen-induced arthritis (CIA) in mice $[40,41]$.

Other fatty acids have only scarcely been studied. Although not yet investigated in relation to human RA, $\alpha$-lipoic acid (ALA) showed promising results in mice, as it inhibited joint inflammation and bone destruction in the CIA model both when administered intra-peritoneally [42] and through diet [43]. Although the mechanisms are unclear, ALA inhibited synovial inflammation in both studies, as well as human osteoclast differentiation in vitro [42] and in mice in vivo [43].

Supporting a possible role for LPA/autotaxin axis in arthritis, inhibition of the LPA receptor inhibits development of disease in the $\mathrm{K} / \mathrm{BxN}$ serum transfer model through effects on cartilage destruction and bone erosions, possibly through inhibition of osteoclast differentiation and activity and promotion of osteoblast differentiation [44]. Similarly, mice lacking autotaxin in the mesenchymal cell compartment had less arthritis on a hTNF- $\alpha$ (tumour necrosis factor- $\alpha$ ) transgenic background, as well as in the CIA model of arthritis and this effect was likely mediated by LPA effects on synovial fibroblasts [25]. Interestingly, preventive oral administration of LPC precursor PC to CIA mice also inhibited severity of CIA, possibly through inhibition of leukocyte-endothelium interactions and nitric oxide (NO) production [45]. These data indicate that the PC/LPC/LPA metabolic pathway might represent a promising target for intervention in RA patients. 


\section{Association with clinical characteristics and intervention studies in $O A$}

Two studies have measured phospholipids in both early OA and late OA patients, with early and late being defined by the Outerbridge classification scale. One of these studies assessed the difference in lipids between early $(n=17)$ and late OA $(n=13)$ and showed that concentrations of 66 phospholipid species were different between these stages and that the total lipid content was higher in late OA compared to early OA [12]. In addition, the PC/LPC ratio was higher in late OA then early OA, possibly indicating a higher activation of $\mathrm{PLA}_{2}$ in early compared to late disease. A metabolomic approach can also be used to classify OA patients based on their metabolite profile. Using this approach, it was found that a group of 80 OA patients was divided into two distinct groups particularly based on the levels of carnitines and its acyl esters acylcarnitines (involved in fatty acid metabolism) in knee SF [46]. The group with lower acylcarnitine levels could be further divided into two subgroups based on their glycophospholipid and sphingomyelin (SM) levels. Although the groups were not correlated to any clinical OA characteristics, these data indicate that OA patients can be divided in metabolically distinct groups. An earlier study by Kosinska et al. did correlate the levels of SM with relevant OA features [47]. All SM species measured in SF were 2.4-fold higher in early OA patients $(n=17)$ compared to controls and twofold higher in late $(n=13)$ versus early OA patients. Early and late OA patients were classified with the Outerbridge score.

Most reports studying lipids and their association with clinical parameters have studied fatty acids, rather than phospholipids or other higher-order lipids. To the best of our knowledge, only one study investigated the association of plasma fatty acid levels with clinical features in OA patients. This study measured fasting plasma levels of fatty acids in 472 individuals, including OA patients, as well as individuals at risk of developing knee OA [48]. N-3 fatty acids, in particular DHA, were inversely correlated with patellofemoral cartilage loss, but not tibiofemoral cartilage loss or synovitis at 30-month follow-up, suggesting a protective effect of this n-3 fatty acid on selected structural findings. The levels of the n-6 fatty acid AA, which is believed to be more pro-inflammatory, were positively correlated with synovitis. In line with this result, in another study on knee OA patients, the AA levels were found to correlate with histologic disease severity [49].

While fish oil or n-3 PUFA supplementation has been intensively studied in RA patients, only a few intervention studies have been performed in OA patient groups. Most of these studies aimed at reducing pain. Forty-seven patients with knee or hip OA, consuming $1200 \mathrm{mg}$ fish oil (total n-3 18\% EPA and 12\% DHA) a day for 12 weeks in a randomized trial, showed no improvement on either visual analogue scale (VAS) for patient self-assessment of pain or the health assessment questionnaire (HAQ) for patient selfassessment of activity [50]. In addition, a double-blind placebo-controlled trial in 86 OA patients failed to detect any benefit from taking either $10 \mathrm{ml}$ cod liver oil (786 mg EPA) or olive oil per day for 24 weeks, next to the regular intake of NSAIDs [51]. By contrast, one study reported the beneficial effects of fish oil in OA patients. A randomized, double-blind multicentre trial investigated the effect of a low dose of $0.45 \mathrm{~g}$ of fish oil (18\% EPA and 12\% DHA) per day versus a high dose of $4.5 \mathrm{~g}$ on pain and function scores in $202 \mathrm{knee}$ OA patients after 2 years [52]. Both groups benefitted from the treatment, although unexpectedly, the group receiving low-dose fish oil benefitted the most, with significantly lower pain scores (WOMAC index) at 18 and 24 months and better function limitation scores after 24 months. No beneficial effects were observed on cartilage volume or bone marrow lesions. It should be mentioned, however, that the low-dose fish oil preparation also contained Sunola oil which includes n-9 monounsaturated oleic acid. Therefore, it is difficult to conclude whether the observed effects were due to the $n-3$ fatty acids alone [53].

Despite the unclear effects of n-3 PUFA on pain in humans, they were shown to reduce radiographic damage in mouse models of OA, both in spontaneous [54] and surgically induced models [55]. Interestingly, their beneficial effect was correlated to a better wound healing, while saturated fatty acids and n-6 fatty acids had opposite effects on OA severity and wound healing [55], suggesting the existence of common mechanisms involved in both processes. Moreover, it suggests a possible benefit of n-3 PUFA on structural damage, which still needs to be investigated in humans.

\section{Effects on RA human tissues in vitro}

Free fatty acids have been described in serum and SF of RA patients and they could affect joint tissues and immune cells involved in disease pathogenesis. Indeed, in vitro treatment of RA synovial 
fibroblast with free fatty acids induced pro-inflammatory cytokines interleukin (IL)-6, IL-8, monocyte chemoattractant protein (MCP)-1 and matrix metalloproteinases (MMPs) and this effect was independent of the length or saturation degree of the fatty acid, but variable between donors [56]. For chondrocytes, lipids isolated from SF could inhibit chondrocyte proliferation [57], while saturated fatty acids induced IL-6 [56]. Endothelial cells only responded to high concentrations of fatty acids such as palmitic acid and linoleic acid, but not oleic acid [56]. Taken together, these data indicate that different cell types respond differently to individual fatty acids and that combinations of fatty acids could have a different effect than the individual components. Moreover, free fatty acids and especially dietary fatty acids can potently influence bone metabolism through regulation of $\mathrm{PGE}_{2}$ and leukotrienes, stimulating bone resorption or prostaglandin-mediated regulation of insulin-like growth factor 1 (IGF-1), a growth factor that stimulated bone formation [58]. The role of these processes in bone metabolism in $\mathrm{RA}$ remain to be investigated.

Besides their effect on joint tissues, fatty acids also have immune modulatory effects. Effects of n-3 PUFA on cells derived from RA patients have been investigated, while much less attention has been given to other fatty acids. N-3 PUFA had an anti-inflammatory effect on cytokine secretion by monocytes derived from RA patients [59], while inhibiting formation of reactive oxygen species [60] and the AA-mediated induction of TNFRI and -II on RA neutrophils [61]. Fatty acids can also affect T cell function [62] and B cell function [63], although the effects of fatty acid type, saturation and length, as well as the specific effects on $\mathrm{T}$ and $\mathrm{B}$ cells from RA patients remain to be addressed.

Some of the phospholipids found in serum or SF have been shown to affect joint tissues and cells thereby potentially contributing to RA. Among these, LPC and LPA have received special attention, as they were described to have potent immune modulatory effects (reviewed in Ref. [64]). For example, LPA has been shown to induce COX2 expression in RA synovial fibroblasts, either alone or in combination with pro-inflammatory cytokines such as IL- $1 \alpha$ or $\beta$ [22], to induce their proliferation and enhance production of IL-6, VEGF, CCL 2 and MMP3, as well as expression of VCAM and migration [65]. Although the effects of LPC on human RA tissues were not yet investigated, LPC was shown to induce COX2 expression in vascular endothelial cells [66] and macrophages [67], which would suggest a possible pro-inflammatory role of this lipid in RA.

Another product of autotaxin found in RA SF, S1P, was also shown to have effects on RA-derived tissues, including stimulation of proliferation of RA synovial fibroblasts [68] and enhancing COX2 and $\mathrm{PGE}_{2}$ production in synovial fibroblasts [68] and chondrocytes [69], or by decreasing aggrecan production by chondrocytes [69] and stimulating osteoblast differentiation [70].

Together, these data indicate a pro-inflammatory effect of the PC/LPC/LPA axis in RA.

\section{Effects on OA human tissues in vitro}

While intervention studies with fatty acid supplementation mainly focussed on reducing pain in patients, the in vitro studies performed focussed predominantly on the effects of fatty acids on OA tissues and the mechanisms underlying these effects. Chondrocytes were most studied in this respect and they were usually stimulated with cytokines believed to have a prominent role in OA. Chondrocytes from knee cartilage explants of OA patients were shown to take up linoleic, oleic and palmitic acid, regardless of whether they were stimulated with TNF- $\alpha$ [71]. Upon oleic acid exposure, TNF- $\alpha-$ stimulated chondrocytes secrete less GAG and downregulate the expression of MMP-1 and COX-2, indicating an anti-inflammatory effect. Palmitic acid had similar effects on GAG release and MMP-1 expression but did not affect $\mathrm{PGE}_{2}$ release or $\mathrm{COX}-2$ expression. By contrast, AA precursor linoleic acid increased the release of $\mathrm{PGE}_{2}$ by TNF- $\alpha$-stimulated chondrocytes. These results suggest that local fatty acid concentrations can contribute to cartilage damage. In another study, the effect of palmitate and oleic acid on chondrocytes of OA patients was investigated in comparison to post-mortem control chondrocytes [72]. The data indicated that IL- $1 \beta$ together with palmitate synergistically increased IL-6 and COX-2 expression, whereas oleic acid did not in both donor types, indicating a rather proinflammatory effect of palmitic acid. In addition, increased apoptotic cell death was observed in chondrocytes stimulated with both IL- $1 \beta$ and palmitate in the post-mortem cartilage. The contrasting effects of palmitic acid on chondrocytes in these two studies could be due to differences in stimulus used to mimic OA-related inflammation in chondrocytes. 


\section{Oxylipins}

\section{Association with clinical characteristics and intervention studies in RA}

It is generally accepted that $\mathrm{PGE}_{2}$ contributes to the disease process in RA [14]. This is primarily based on studies in mouse models of arthritis, as well as on intervention studies in humans. Deficiencies in enzymes involved in $\mathrm{PGE}_{2}$ generation, such as CPLA 2 [73], COX-1 [74] and COX-2 [75] or mPGES-1 [76], as well as blockers of PGE $_{2}$ receptors [77] were associated with diminished disease in mouse models of arthritis. Moreover, intervention studies conducted using pharmacological inhibitors, which have also been used in humans, indicated that $\mathrm{PGE}_{2}$ contributes to pain in antibody-induced models of arthritis, especially in the inflammatory phase of the disease [78,79]. Taken together, these data indicate that $\mathrm{PGE}_{2}$ plays a role in this disease. Intriguingly, however, it was also suggested that $\mathrm{PGE}_{2}$ has a dual role in arthritis, being pro-inflammatory in the induction phase, while also contributing to disease resolution, by inducing the pro-resolving lipid mediator $\mathrm{LXA}_{4}$ during the later phases [80]. $\mathrm{LXA}_{4}$ has also been shown to have anti-inflammatory and pro-resolving properties in other murine arthritis models, such as zymosan-induced arthritis [81].

In humans, most evidence for a deleterious contribution of $\mathrm{PGE}_{2}$ to pain was obtained from studies investigating the effect of NSAIDS in RA (reviewed in Ref. [82]). The involvement of $\mathrm{PGE}_{2}$ in inflammation in RA is less clear, although some studies indicate that local administration of corticosteroids in knee RA also diminishes swelling besides pain and this is parallelled by a reduction in MPGES-1, COX-1 and COX-2 expression, as well as less $\mathrm{PGE}_{2}$ production [83]. In line with this, randomized control trials indicate that drugs aimed at blocking COX activity also affect the number of swollen joints [84]. Because COX is involved in the generation of several lipid species, including anti-inflammatory ones, such as $\mathrm{PGJ}_{2}$ or the pro-resolving E series resolvins, it would be highly interesting to test the clinical efficacy of inhibitors of mPGES or antagonists of $\mathrm{PGE}_{2}$ receptors, which would be expected to modulate more specifically $\mathrm{PGE}_{2}$-mediated effects.

Besides prostaglandins, leukotrienes, especially $\mathrm{LTB}_{4}$, could also play a role in RA. Serum levels of $\mathrm{LTB}_{4}$ were associated with higher disease activity [85], while SF levels of LTB 4 were correlated with inflammatory markers (e.g. cellular infiltrate) in RA patients [86]. However, these data should be interpreted carefully, as 5S,12S-diHETE, a less active isomer of $\mathrm{LTB}_{4}$, was also described in SF of RA patients [11]. It is difficult to distinguish these two lipids with enzyme-linked immunosorbent assay ELISA or high-performance liquid chromatography (HPLC) techniques (own unpublished data). Murine studies provide evidence for the role of $\mathrm{LTB}_{4}$ in RA, wherein 5-LOX or LTA 4 hydrolase deficiency prevented the development of antibody-induced arthritis and this could be restored by transfer of neutrophils capable of secreting $\mathrm{LTB}_{4}$, thus indicating a role for this lipid and neutrophils in this model [87]. Similarly, the absence of the $\mathrm{LTB}_{4}$ receptor BLT1, especially on neutrophils, also resulted in less arthritis in the K/BxN and CIA models [88,89]. Data in RA patients are less clear. Zileuton, a 5-LOX inhibitor, did not decrease joint tenderness and pain after a 4 -week treatment in patients with active RA. However, there was a nonsignificant decline in the number of joints effected [90].

\section{Association with clinical characteristics and intervention studies in $O A$}

Studies investigating the role of oxylipins in OA patients are scarce. In a recent study, plasma levels of $\mathrm{PGE}_{2}$ and 15 -HETE, as well as TNF- $\alpha$, IL- $1 \beta$ and COX-2 expression in peripheral blood leukocytes (PBL) were investigated in three separate cohorts of knee OA patients [28]. Higher levels of plasma $\mathrm{PGE}_{2}$ and 15-HETE were associated with the presence of knee OA, while a higher expression of TNF $\alpha$, IL-1 $\beta$ and COX-2 in PBL at baseline predicted more rapid progression of joint space narrowing 24 months later.

In a recent systematic review, the efficacy of pharmacological interventions for knee OA was investigated [91]. In total, 137 randomized controlled trials were summarized. Treatments included COX inhibitors such as diclofenac, ibuprofen, naproxen and celecoxib. All treatments were more efficient than placebo in controlling pain, while intra-articular treatments were superior to oral treatments. Regarding function and stiffness, all treatments with COX inhibitors were more effective than oral placebo, thus indicating a possible beneficial effect of these pharmacological 
agents in OA. Another enzyme involved in the generation of lipid mediators is 5-LOX. The safety of a natural 5-LOX inhibitor was evaluated in 75 OA patients [92]. The inhibitor showed pain reduction in the treated group compared to placebo; however, effects on structural damage in patients remain to be defined.

A third approach is to inhibit both COX and 5-LOX enzymes, as several studies now showed that the dual COX/5-LOX inhibitor licofelone is equally effective in diminishing pain as the COX inhibitor naproxen, but has less gastrointestinal-related adverse effects [93]. Moreover, in one study with 355 OA patients, licofelone was more efficient than naproxen in reducing cartilage volume loss in the global joint and lateral compartment at 6,12 and 24 months [94]. However, the effects of this compound on $\mathrm{OA}$ structural damage compared to placebo were not yet investigated in humans. Nevertheless, two studies on OA models in dogs indicated less cartilage lesions and decreased levels of $\mathrm{PGE}_{2}, \mathrm{LTB}_{4}$, collagenase 1 and IL-1 $\beta$ in the joint in the treated group [95], as well as reduced size of cartilage lesions and development of osteophytes [96]. These findings are supported by in vitro studies, in which IL-1 $\beta$-treated chondrocytes displayed decreased MMP-13 production upon treatment with licofelone [97].

\section{Effects on RA human tissues in vitro}

Several studies investigated the effect of prostaglandins, especially $\mathrm{PGE}_{2}$, on tissues derived from RA patients. In line with what was observed in vivo, $\mathrm{PGE}_{2}$ can display both pro- and anti-inflammatory effects. PGE 2 has been shown to upregulate IL-6 and mPGES in RA synovial fibroblasts $[98,99]$ and IL-6 in chondrocytes [100], while inhibiting RA fibroblast growth [101] and MMP-1 expression (JI 2009; 15:1328) as well as osteoclast development [101]. Besides immune cells, PGE 2 can have proinflammatory effects also on dendritic cells, by inducing IL-23 production [102] and antiinflammatory effects on monocytes and macrophages [103] by reducing the p40 subunit of IL-12 and IL-23. These data suggest that the inhibition of $\mathrm{PGE}_{2}$ in RA should be carefully considered. For 15-deoxy-PGJ 2 , predominantly anti-inflammatory effects were shown, such as downregulation of IL-6 [100] and induction of apoptosis in chondrocytes [104], as well as inhibition of TNF $\alpha$-induced MMP-13 production in RA synovial fibroblasts [105].

In vitro studies indicate that leukotriene B4 has a pro-inflammatory effect on RA tissues. $\mathrm{LTB}_{4}$ induced TNF $\alpha$ and IL-1 $\beta$ in RA synovial fibroblasts [106] and was capable of inducing osteoclast differentiation [107].

The effect of anti-inflammatory lipid mediators was much less studied. Intriguingly, one study indicated that 15-HETE, a derivative of 15-LOX, could upregulate MMP-2 in RA synovial fibroblasts [108], suggesting a possible pro-inflammatory role of this lipid in RA.

\section{Effects on human tissues in vitro $O A$}

Several studies investigated the effect of oxylipins on OA tissues in vitro. 15-LOX products as well as PGD(COX product) dose dependently decreased IL- $1 \beta$-induced MMP- 1 and MMP-13 production by chondrocytes isolated from OA cartilage [109], thus suggesting a beneficial role for these lipids in OA. By contrast, the 5 -LOX product $\mathrm{LTB}_{4}$ was shown to increase IL- $1 \beta$ secretion by OA synovial membranes [110], while increasing osteocalcin secretion by OA osteoblasts [111], thus indicating that $\mathrm{LTB}_{4}$ might contribute to inflammation and structural damage in OA patients.

$\mathrm{PGE}_{2}$ is the most studied oxylipin in the context of OA and has been shown to induce IL-6, VEGF and M-SCF production by human OA synovial fibroblasts [112] and to have deleterious effects on OA chondrocytes. On articular cartilage of $\mathrm{OA}$ patients, $\mathrm{PGE}_{2}$ inhibits proteoglycan release and stimulated MMP-13 production and collagen type II breakdown via engagement of EP receptors. Blocking the EP4 receptor could inverse these effects, thus indicating it as a potential therapeutic target, more specific than COX inhibitors [113]. However, it was also shown that $\mathrm{PGE}_{2}$ at concentrations lower than found in SF are important in maintaining normal chondrocyte phenotype [114]. Taken together, these data indicate that future studies on $\mathrm{PGE}_{2}$ and its signalling pathways are required to fully understand its contribution to OA. 


\section{Practice points}

- Lipid mediators such as prostaglandins and leukotrienes are increased in synovial fluid of both RA and OA patients.

- Anti-inflammatory lipid mediators were detected in RA synovial fluid, thus indicating activation of regulatory mechanisms.

- $\mathrm{PGE}_{2}$ can have both deleterious and beneficial effects.

- COX is involved in pain perception in RA and OA.

- Targeting COX and LOX enzymes can affect both pro- and anti-inflammatory/pro-resolving lipid synthesis.

- N-3 PUFA supplementation is suggested to have (small) beneficial effects in RA patients.

\section{Research agenda}

- The effects of n-3 PUFA supplementation in RA and OA should be further investigated in high-quality randomized controlled trials.

- Therapeutical interventions aimed at inhibiting lipid mediator receptors rather than the enzymes involved in their generation should be studied in humans.

- The effects of $\mathrm{PGE}_{2}$ in inflammation in RA need further investigation.

- The involvement of COX and LOX enzymes and their lipid products in development or severity of OA needs further pre-clinical investigation.

- The overall effect of anti-inflammatory/pro-resolving lipids on OA and RA tissue needs to be addressed.

\section{Summary}

Fatty acids, phospholipids and oxylipins can be detected in RA and OA patients. Most evidence suggests the activation of the COX/PGE 2 and autotaxin/LPA axes in RA and the COX/PGE 2 and LOX pathways in OA. Inhibition of the COX pathway is beneficial for pain in both diseases, while the effects of inhibition of the LOX pathway are unclear. Whether and how lipids generated by these two pathways are involved in structural damage and inflammation in these diseases is still under investigation. Moreover, the role of anti-inflammatory (oxy)lipids in RA and OA must be studied.

\section{Acknowledgements}

This work was funded by the Dutch Arthritis Foundation (long-term research project, LLP-24), the Netherlands Organisation for Scientific Research (Grant 819.02.003 and VICI scheme) and the IMI JU funded project BeTheCure. We would like to thank Mathieu Visser for assistance with graphical design.

\section{References}

[1] Yusuf E, Kortekaas MC, Watt I, et al. Do knee abnormalities visualised on MRI explain knee pain in knee osteoarthritis? A systematic review. Annals of the Rheumatic Diseases 2011;70:60-7.

[2] Berenbaum F. Osteoarthritis as an inflammatory disease (osteoarthritis is not osteoarthrosis!). Osteoarthritis and Cartilage 2013;21:16-21.

[3] Boyer JF, Gourraud PA, Cantagrel A, et al. Traditional cardiovascular risk factors in rheumatoid arthritis: a meta-analysis. Joint Bone Spine 2011;78:179-83.

[4] de MW, van der Kraan PM, van den Berg WB, et al. High systemic levels of low-density lipoprotein cholesterol: fuel to the flames in inflammatory osteoarthritis?. Oxford: Rheumatology; 2015. 
[5] Quehenberger O, Armando AM, Brown AH, et al. Lipidomics reveals a remarkable diversity of lipids in human plasma. Journal of Lipid Research 2010;51:3299-305.

[6] Young SP, Kapoor SR, Viant MR, et al. The impact of inflammation on metabolomic profiles in patients with arthritis. Arthritis \& Rheumatism 2013;65:2015-23.

[7] Ormseth MJ, Swift LL, Fazio S, et al. Free fatty acids are associated with insulin resistance but not coronary artery atherosclerosis in rheumatoid arthritis. Atherosclerosis 2011;219:869-74.

[8] Fuchs B, Bondzio A, Wagner U, et al. Phospholipid compositions of sera and synovial fluids from dog, human and horse: a comparison by 31P-NMR and MALDI-TOF MS. Journal of Animal Physiology and Animal Nutricion 2009;93:410-22.

[9] Fuchs B, Schiller J, Wagner U, et al. The phosphatidylcholine/lysophosphatidylcholine ratio in human plasma is an indicator of the severity of rheumatoid arthritis: investigations by 31P NMR and MALDI-TOF MS. Clinical Biochemistry 2005;38:925-33.

[10] Moghaddami M, Ranieri E, James M, et al. Prostaglandin D(2) in inflammatory arthritis and its relation with synovial fluid dendritic cells. Mediators of Inflammation 2013;2013:329494.

*[11] Giera M, Ioan-Facsinay A, Toes R, et al. Lipid and lipid mediator profiling of human synovial fluid in rheumatoid arthritis patients by means of LC-MS/MS. Biochimica et Biophysica Acta 2012;1821:1415-24.

[12] Kosinska MK, Liebisch G, Lochnit G, et al. A lipidomic study of phospholipid classes and species in human synovial fluid. Arthritis \& Rheumatism 2013;65:2323-33.

*[13] Kosinska MK, Ludwig TE, Liebisch G, et al. Articular joint lubricants during osteoarthritis and rheumatoid arthritis display altered levels and molecular species. PLoS One 2015;10:e0125192.

[14] Korotkova M, Jakobsson PJ. Persisting eicosanoid pathways in rheumatic diseases. Nature Reviews Rheumatology 2014; 10:229-41.

[15] Hishinuma T, Nakamura H, Sawai T, et al. Microdetermination of prostaglandin E2 in joint fluid in rheumatoid arthritis patients using gas chromatography/selected ion monitoring. Prostaglandins \& Other Lipid Mediators 1999;58:179-86.

[16] Pruzanski W, Vadas P, Kim J, et al. Phospholipase A2 activity associated with synovial fluid cells. The Journal of Rheumatology 1988;15:791-4.

[17] Seilhamer JJ, Plant S, Pruzanski W, et al. Multiple forms of phospholipase A2 in arthritic synovial fluid. The Journal of Biochemistry 1989;106:38-42.

[18] Westman M, Korotkova M, af KE, et al. Expression of microsomal prostaglandin E synthase 1 in rheumatoid arthritis synovium. Arthritis \& Rheumatism 2004;50:1774-80.

[19] Murakami M, Nakashima K, Kamei D, et al. Cellular prostaglandin E2 production by membrane-bound prostaglandin E synthase-2 via both cyclooxygenases-1 and -2. The Journal of Biological Chemistry 2003;278:37937-47.

[20] Shao XT, Feng L, Gu LJ, et al. Expression of interleukin-18, IL-18BP, and IL-18R in serum, synovial fluid, and synovial tissue in patients with rheumatoid arthritis. Clinical and Experimental Medicine 2009;9:215-21.

[21] Serhan CN, Chiang N, Dalli J. The resolution code of acute inflammation: novel pro-resolving lipid mediators in resolution. Seminars in Immunology 2015;27:200-15.

[22] Nochi H, Tomura H, Tobo M, et al. Stimulatory role of lysophosphatidic acid in cyclooxygenase-2 induction by synovial fluid of patients with rheumatoid arthritis in fibroblast-like synovial cells. The Journal of Immunology 2008;181:5111-9.

[23] Limaye V, Xia P, Hahn C, et al. Chronic increases in sphingosine kinase-1 activity induce a pro-inflammatory, proangiogenic phenotype in endothelial cells. Cellular and Molecular Biology Letters 2009;14:424-41.

[24] Song HY, Lee MJ, Kim MY, et al. Lysophosphatidic acid mediates migration of human mesenchymal stem cells stimulated by synovial fluid of patients with rheumatoid arthritis. Biochimica et Biophysica Acta 2010;1801:23-30.

[25] Nikitopoulou I, Oikonomou N, Karouzakis E, et al. Autotaxin expression from synovial fibroblasts is essential for the pathogenesis of modeled arthritis. The Journal of Experimental Medicine 2012;209:925-33.

[26] Castro-Perez JM, Kamphorst J, DeGroot J, et al. Comprehensive LC-MS E lipidomic analysis using a shotgun approach and its application to biomarker detection and identification in osteoarthritis patients. Journal of Proteome Research 2010;9: 2377-89.

[27] Basu S, Whiteman M, Mattey DL, et al. Raised levels of F(2)-isoprostanes and prostaglandin F(2alpha) in different rheumatic diseases. Annals of the Rheumatic Diseases 2001;60:627-31.

*[28] Attur M, Krasnokutsky S, Statnikov A, et al. Low-grade inflammation in symptomatic knee osteoarthritis: prognostic value of inflammatory plasma lipids and peripheral blood leukocyte biomarkers. Arthritis \& Rheumatology 2015;67: 2905-15.

[29] Rae SA, Davidson EM, Smith MJ. Leukotriene B4, an inflammatory mediator in gout. Lancet 1982:1122-4.

[30] Lee TH, Hoover RL, Williams JD, et al. Effect of dietary enrichment with eicosapentaenoic and docosahexaenoic acids on in vitro neutrophil and monocyte leukotriene generation and neutrophil function. The New England Journal of Medicine 1985;312:1217-24.

*[31] Miles EA, Calder PC. Influence of marine n-3 polyunsaturated fatty acids on immune function and a systematic review of their effects on clinical outcomes in rheumatoid arthritis. British Journal of Nutrition 2012;107(Suppl. 2):S171-84.

[32] Vidgren HM, Agren JJ, Schwab U, et al. Incorporation of n-3 fatty acids into plasma lipid fractions, and erythrocyte membranes and platelets during dietary supplementation with fish, fish oil, and docosahexaenoic acid-rich oil among healthy young men. Lipids 1997;32:697-705.

[33] Skarke C, Alamuddin N, Lawson JA, et al. Bioactive products formed in humans from fish oils. The Journal of Lipid Research 2015;56:1808-20.

[34] Park Y, Lee A, Shim SC, et al. Effect of n-3 polyunsaturated fatty acid supplementation in patients with rheumatoid arthritis: a 16-week randomized, double-blind, placebo-controlled, parallel-design multicenter study in Korea. The Journal of Nutritional Biochemistry 2013;24:1367-72.

[35] Dawczynski C, Hackermeier U, Viehweger M, et al. Incorporation of n-3 PUFA and gamma-linolenic acid in blood lipids and red blood cell lipids together with their influence on disease activity in patients with chronic inflammatory arthritis-a randomized controlled human intervention trial. Lipids in Health and Disease 2011;10:130.

[36] Proudman SM, James MJ, Spargo LD, et al. Fish oil in recent onset rheumatoid arthritis: a randomised, double-blind controlled trial within algorithm-based drug use. Annals of the Rheumatic Diseases 2015;74:89-95. 
*[37] Di Giuseppe D, Crippa A, Orsini N, et al. Fish consumption and risk of rheumatoid arthritis: a dose-response metaanalysis. Arthritis Research \& Therapy 2014;16:446.

[38] Di Giuseppe D, Wallin A, Bottai M, et al. Long-term intake of dietary long-chain n-3 polyunsaturated fatty acids and risk of rheumatoid arthritis: a prospective cohort study of women. Annals of the Rheumatic Diseases 2014;73:1949-53.

[39] Gan RW, Young KA, Zerbe GO, et al. Lower omega-3 fatty acids are associated with the presence of anti-cyclic citrullinated peptide autoantibodies in a population at risk for future rheumatoid arthritis: a nested case-control study. Rheumatology Oxford 2015;55:367-76.

[40] Leslie CA, Gonnerman WA, Ullman MD, et al. Dietary fish oil modulates macrophage fatty acids and decreases arthritis susceptibility in mice. The Journal of Experimental Medicine 1985;162:1336-49.

[41] Ierna M, Kerr A, Scales H, et al. Supplementation of diet with krill oil protects against experimental rheumatoid arthritis. BMC Musculoskeletal Disorders 2010;11:136.

[42] Lee EY, Lee CK, Lee KU, et al. Alpha-lipoic acid suppresses the development of collagen-induced arthritis and protects against bone destruction in mice. Rheumatology International 2007;27:225-33.

[43] Hah YS, Sung MJ, Lim HS, et al. Dietary alpha lipoic acid supplementation prevents synovial inflammation and bone destruction in collagen-induced arthritic mice. Rheumatology International Int 2011;31:1583-90.

[44] Orosa B, Garcia S, Martinez P, et al. Lysophosphatidic acid receptor inhibition as a new multipronged treatment for rheumatoid arthritis. Annals of the Rheumatic Diseases 2014;73:298-305.

[45] Eros G, Ibrahim S, Siebert N, et al. Oral phosphatidylcholine pretreatment alleviates the signs of experimental rheumatoid arthritis. Arthritis Research \& Therapy 2009;11:R43.

[46] Zhang W, Likhodii S, Zhang Y, et al. Classification of osteoarthritis phenotypes by metabolomics analysis. BMJ Open 2014; 4:e006286.

[47] Kosinska MK, Liebisch G, Lochnit G, et al. Sphingolipids in human synovial fluid-a lipidomic study. PLoS One 2014;9:e91769.

[48] Baker KR, Matthan NR, Lichtenstein AH, et al. Association of plasma n-6 and n-3 polyunsaturated fatty acids with synovitis in the knee: the MOST study. Osteoarthritis and Cartilage 2012;20:382-7.

[49] Lippiello L, Walsh T, Fienhold M. The association of lipid abnormalities with tissue pathology in human osteoarthritic articular cartilage. Metabolism 1991;40:571-6.

[50] Zawadzki M, Janosch C, Szechinski J. Perna canaliculus lipid complex PCSO-524 demonstrated pain relief for osteoarthritis patients benchmarked against fish oil, a randomized trial, without placebo control. Marine drugs 2013;11: $1920-35$.

[51] Stammers T, Sibbald B, Freeling P. Efficacy of cod liver oil as an adjunct to non-steroidal anti-inflammatory drug treatment in the management of osteoarthritis in general practice. Annals of the Rheumatic Diseases 1992;51:128-9.

*[52] Hill CL, March LM, Aitken D, et al. Fish oil in knee osteoarthritis: a randomised clinical trial of low dose versus high dose. Annals of the Rheumatic Diseases 2015. http://dx.doi.org/10.1136/annrheumdis-2014-207169.

[53] Felson DT, Bischoff-Ferrari HA. Dietary fatty acids for the treatment of OA, including fish oil. Annals of the Rheumatic Diseases 2016;75:1-2.

[54] Knott L, Avery NC, Hollander AP, et al. Regulation of osteoarthritis by omega-3 (n-3) polyunsaturated fatty acids in a naturally occurring model of disease. Osteoarthritis and Cartilage 2011;19:1150-7.

[55] Wu CL, Jain D, McNeill JN, et al. Dietary fatty acid content regulates wound repair and the pathogenesis of osteoarthritis following joint injury. Annals of the Rheumatic Diseases 2015;74:2076-83.

[56] Frommer KW, Schaffler A, Rehart S, et al. Free fatty acids: potential proinflammatory mediators in rheumatic diseases. Annals of the Rheumatic Diseases 2015;74:303-10.

[57] Colantuoni G, Quintero M, Panasyuk A, et al. Do arachidonic acid and its metabolites, secreted by rheumatoid and osteoarthritic synovial tissue, account for the strong inhibition of DNA synthesis in cultured human articular chondrocytes? A novel approach to the mechanism of tissue damage. Joint Bone Spine 2005;72:533-9.

[58] Watkins BA, Lippman HE, Le BL, et al. Bioactive fatty acids: role in bone biology and bone cell function. Progress in Lipid Research 2001;40:125-48.

[59] Kremer JM, Lawrence DA, Jubiz W, et al. Dietary fish oil and olive oil supplementation in patients with rheumatoid arthritis. Clinical and immunologic effects. Arthritis \& Rheumatism 1990;33:810-20.

[60] Magaro M, Altomonte L, Zoli A, et al. Influence of diet with different lipid composition on neutrophil chemiluminescence and disease activity in patients with rheumatoid arthritis. Annals of the Rheumatic Diseases 1988;47:793-6.

[61] Moghaddami N, Irvine J, Gao X, et al. Novel action of n-3 polyunsaturated fatty acids: inhibition of arachidonic acidinduced increase in tumor necrosis factor receptor expression on neutrophils and a role for proteases. Arthritis \& Rheumatism 2007:56:799-808.

[62] de Jong AJ, Kloppenburg M, Toes RE, et al. Fatty acids, lipid mediators, and T-cell function. Frontiers in Immunology 2014; $5: 483$.

[63] Shaikh SR, Teague H. N-3 fatty acids and membrane microdomains: from model membranes to lymphocyte function. Prostaglandins, Leukotrienes \& Essential Fatty Acids 2012;87:205-8.

[64] Sevastou I, Kaffe E, Mouratis MA, et al. Lysoglycerophospholipids in chronic inflammatory disorders: the PLA(2)/LPC and ATX/LPA axes. Biochimica et Biophysica Acta 2013;1831:42-60.

[65] Miyabe Y, Miyabe C, Iwai Y, et al. Activation of fibroblast-like synoviocytes derived from rheumatoid arthritis via lysophosphatidic acid-lysophosphatidic acid receptor 1 cascade. Arthritis Research \& Therapy 2014;16:461.

[66] Rikitake Y, Hirata K, Kawashima S, et al. Signaling mechanism underlying COX-2 induction by lysophosphatidylcholine. Biochemical and Biophysical Research Communications 2001;281:1291-7.

[67] Ruiperez V, Casas J, Balboa MA, et al. Group V phospholipase A2-derived lysophosphatidylcholine mediates cyclooxygenase-2 induction in lipopolysaccharide-stimulated macrophages. The Journal of Immunology 2007;179: $631-8$.

[68] Kitano M, Hla T, Sekiguchi M, et al. Sphingosine 1-phosphate/sphingosine 1-phosphate receptor 1 signaling in rheumatoid synovium: regulation of synovial proliferation and inflammatory gene expression. Arthritis \& Rheumatism 2006; 54:742-53. 
[69] Masuko K, Murata M, Nakamura H, et al. Sphingosine-1-phosphate attenuates proteoglycan aggrecan expression via production of prostaglandin E2 from human articular chondrocytes. BMC Musculoskeletal Disorders 2007;8:29.

[70] Sato C, Iwasaki T, Kitano S, et al. Sphingosine 1-phosphate receptor activation enhances BMP-2-induced osteoblast differentiation. Biochemical and Biophysical Research Communications 2012;423:200-5.

[71] Bastiaansen-Jenniskens YM, Siawash M, van de Lest CH, et al. Monounsaturated and saturated, but not n-6 polyunsaturated fatty acids decrease cartilage destruction under inflammatory conditions: a preliminary study. Cartilage 2013;4:321-8.

[72] Alvarez-Garcia O, Rogers NH, Smith RG, et al. Palmitate has proapoptotic and proinflammatory effects on articular cartilage and synergizes with interleukin-1. Arthritis \& Rheumatology 2014;66:1779-88.

[73] Raichel L, Berger S, Hadad N, et al. Reduction of cPLA2alpha overexpression: an efficient anti-inflammatory therapy for collagen-induced arthritis. European Journal of Immunology 2008;38:2905-15.

[74] Chen M, Boilard E, Nigrovic PA, et al. Predominance of cyclooxygenase 1 over cyclooxygenase 2 in the generation of proinflammatory prostaglandins in autoantibody-driven K/BxN serum-transfer arthritis. Arthritis \& Rheumatism 2008;58: 1354-65.

[75] Myers LK, Kang AH, Postlethwaite AE, et al. The genetic ablation of cyclooxygenase 2 prevents the development of autoimmune arthritis. Arthritis \& Rheumatism 2000;43:2687-93.

[76] Trebino CE, Stock JL, Gibbons CP, et al. Impaired inflammatory and pain responses in mice lacking an inducible prostaglandin E synthase. Proceedings of the National Academy of Sciences of the United Stated of America 2003;100: 9044-9.

[77] Clark P, Rowland SE, Denis D, et al. MF498 [N-\{[4-(5,9-Diethoxy-6-oxo-6,8-dihydro-7H-pyrrolo[3,4-g]quinolin-7-yl)-3methylbe nzyl]sulfonyl\}-2-(2-methoxyphenyl)acetamide], a selective E prostanoid receptor 4 antagonist, relieves joint inflammation and pain in rodent models of rheumatoid and osteoarthritis. Journal of Pharmacology and Experimental Therapeutics 2008;325:425-34.

[78] Christianson CA, Corr M, Firestein GS, et al. Characterization of the acute and persistent pain state present in $\mathrm{K} / \mathrm{BxN}$ serum transfer arthritis. Pain 2010;151:394-403.

[79] Bas DB, Su J, Sandor K, et al. Collagen antibody-induced arthritis evokes persistent pain with spinal glial involvement and transient prostaglandin dependency. Arthritis \& Rheumatism 2012;64:3886-96.

*[80] Chan MM, Moore AR. Resolution of inflammation in murine autoimmune arthritis is disrupted by cyclooxygenase-2 inhibition and restored by prostaglandin E2-mediated lipoxin A4 production. The Journal of Immunology 2010;184: $6418-26$.

[81] Conte FP, Menezes-de-Lima Jr O, Verri Jr WA, et al. Lipoxin A(4) attenuates zymosan-induced arthritis by modulating endothelin-1 and its effects. British Journal of Pharmacology 2010;161:911-24.

*[82] Martel-Pelletier J, Pelletier JP, Fahmi H. Cyclooxygenase-2 and prostaglandins in articular tissues. Seminars in Arthritis and Rheumatism 2003;33:155-67.

[83] Korotkova M, Westman M, Gheorghe KR, et al. Effects of antirheumatic treatments on the prostaglandin E2 biosynthetic pathway. Arthritis \& Rheumatism 2005;52:3439-47.

[84] Garner S, Fidan D, Frankish R, et al. Celecoxib for rheumatoid arthritis. Cochrane Database of Systematic Reviews 2002: CD003831.

[85] Gursel T, Firat S, Ercan ZS. Increased serum leukotriene B4 level in the active stage of rheumatoid arthritis in children. Prostaglandins, Leukotrienes \& Essential Fatty Acids 1997;56:205-7.

[86] Ahmadzadeh N, Shingu M, Nobunaga M, et al. Relationship between leukotriene B4 and immunological parameters in rheumatoid synovial fluids. Inflammation 1991;15:497-503.

[87] Chen M, Lam BK, Kanaoka Y, et al. Neutrophil-derived leukotriene B4 is required for inflammatory arthritis. The Journal of Experimental Medicine 2006;203:837-42.

[88] Chou RC, Kim ND, Sadik CD, et al. Lipid-cytokine-chemokine cascade drives neutrophil recruitment in a murine model of inflammatory arthritis. Immunity 2010;33:266-78.

[89] Shao WH, Del PA, Bock CB, et al. Targeted disruption of leukotriene B4 receptors BLT1 and BLT2: a critical role for BLT1 in collagen-induced arthritis in mice. The Journal of Immunology 2006;176:6254-61.

[90] Weinblatt ME, Kremer JM, Coblyn JS, et al. Zileuton, a 5-lipoxygenase inhibitor in rheumatoid arthritis. The Journal of Rheumatology 1992;19:1537-41.

*[91] Bannuru RR, Schmid CH, Kent DM, et al. Comparative effectiveness of pharmacologic interventions for knee osteoarthritis: a systematic review and network meta-analysis. Annals of Internal Medicine 2015;162:46-54.

[92] Sengupta K, Alluri KV, Satish AR, et al. A double blind, randomized, placebo controlled study of the efficacy and safety of 5-Loxin for treatment of osteoarthritis of the knee. Arthritis Research \& Therapy 2008;10:R85.

[93] Cicero AF, Laghi L. Activity and potential role of licofelone in the management of osteoarthritis. Journal of Clinical Interventions in Aging 2007;2:73-9.

*[94] Raynauld JP, Martel-Pelletier J, Bias P, et al. Protective effects of licofelone, a 5-lipoxygenase and cyclo-oxygenase inhibitor, versus naproxen on cartilage loss in knee osteoarthritis: a first multicentre clinical trial using quantitative MRI. Annals of the Rheumatic Diseases 2009;68:938-47.

[95] Jovanovic DV, Fernandes JC, Martel-Pelletier J, et al. In vivo dual inhibition of cyclooxygenase and lipoxygenase by ML3000 reduces the progression of experimental osteoarthritis: suppression of collagenase 1 and interleukin-1beta synthesis. Arthritis \& Rheumatism 2001;44:2320-30.

[96] Moreau M, Boileau C, Martel-Pelletier J, et al. Licofelone reduces progression of structural changes in a canine model of osteoarthritis under curative conditions: effect on protease expression and activity. The Journal of Rheumatology 2006; 33:1176-83.

[97] Boileau C, Pelletier JP, Tardif G, et al. The regulation of human MMP-13 by licofelone, an inhibitor of cyclo-oxygenases and 5-lipoxygenase, in human osteoarthritic chondrocytes is mediated by the inhibition of the p38 MAP kinase signalling pathway. Annals of the Rheumatic Diseases 2005;64:891-8.

[98] Sommerfelt RM, Feuerherm AJ, Skuland T, et al. Cytosolic phospholipase A2 modulates TLR2 signaling in synoviocytes. PLoS One 2015;10:e0119088. 
[99] Kojima F, Naraba H, Sasaki Y, et al. Prostaglandin E2 is an enhancer of interleukin-1beta-induced expression of membrane-associated prostaglandin E synthase in rheumatoid synovial fibroblasts. Arthritis \& Rheumatism 2003;48: 2819-28.

[100] Wang P, Zhu F, Konstantopoulos K. Interleukin-6 synthesis in human chondrocytes is regulated via the antagonistic actions of prostaglandin (PG)E2 and 15-deoxy-Delta(12,14)-PGJ2. PLoS One 2011;6:e27630.

[101] Shibata-Nozaki T, Ito H, Mitomi H, et al. Endogenous prostaglandin E2 inhibits aberrant overgrowth of rheumatoid synovial tissue and the development of osteoclast activity through EP4 receptor. Arthritis \& Rheumatism 2011;63: 2595-605.

[102] Sheibanie AF, Tadmori I, Jing H, et al. Prostaglandin E2 induces IL-23 production in bone marrow-derived dendritic cells. Federation of American Societies for Experimental Biology Journal 2004;18:1318-20.

[103] Klein-Wieringa IR, Andersen SN, Kwekkeboom JC, et al. Adipocytes modulate the phenotype of human macrophages through secreted lipids. The Journal of Immunology 2013;191:1356-63.

[104] Shan ZZ, Masuko-Hongo K, Dai SM, et al. A potential role of 15-deoxy-delta(12,14)-prostaglandin J2 for induction of human articular chondrocyte apoptosis in arthritis. The Journal of Biological Chemistry 2004;279:37939-50.

[105] Lin TH, Tang CH, Wu K, et al. 15-deoxy-Delta(12,14) -prostaglandin-J2 and ciglitazone inhibit TNF-alpha-induced matrix metalloproteinase 13 production via the antagonism of NF-kappaB activation in human synovial fibroblasts. Journal of Cellular Physiology 2011;226:3242-50.

[106] Xu S, Lu H, Lin J, et al. Regulation of TNFalpha and IL1beta in rheumatoid arthritis synovial fibroblasts by leukotriene B4. Rheumatology International 2010;30:1183-9.

[107] Chen ZK, Lv HS, Jiang J. LTB4 can stimulate human osteoclast differentiation dependent of RANKL. Artificial Cells, Blood Substitutes, and Immobilization Biotechnology 2010;38:52-6.

[108] Wu MY, Lin TH, Chiu YC, et al. Involvement of 15-lipoxygenase in the inflammatory arthritis. Journal of Cellular Biochemistry 2012;113:2279-89.

[109] Chabane N, Zayed N, Benderdour M, et al. Human articular chondrocytes express 15-lipoxygenase-1 and -2: potential role in osteoarthritis. Arthritis Research \& Therapy 2009;11:R44.

[110] Marcouiller P, Pelletier JP, Guevremont M, et al. Leukotriene and prostaglandin synthesis pathways in osteoarthritic synovial membranes: regulating factors for interleukin 1beta synthesis. The Journal of Rheumatology 2005;32:704-12.

[111] Paredes Y, Massicotte F, Pelletier JP, et al. Study of the role of leukotriene B( 4 in abnormal function of human subchondral osteoarthritis osteoblasts: effects of cyclooxygenase and/or 5-lipoxygenase inhibition. Arthritis \& Rheumatism 2002;46: 1804-12.

[112] Inoue H, Takamori M, Shimoyama Y, et al. Regulation by PGE2 of the production of interleukin-6, macrophage colony stimulating factor, and vascular endothelial growth factor in human synovial fibroblasts. British Journal of Pharmacology 2002;136:287-95.

[113] Attur M, Al-Mussawir HE, Patel J, et al. Prostaglandin E2 exerts catabolic effects in osteoarthritis cartilage: evidence for signaling via the EP4 receptor. The Journal of Immunology 2008;181:5082-8.

[114] Tchetina EV, Di Battista JA, Zukor DJ, et al. Prostaglandin PGE2 at very low concentrations suppresses collagen cleavage in cultured human osteoarthritic articular cartilage: this involves a decrease in expression of proinflammatory genes, collagenases and COL10A1, a gene linked to chondrocyte hypertrophy. Arthritis Research \& Therapy 2007;9:R75. 